\title{
Intensive psychotherapy and case management for Karen refugees with major depression in primary care: a pragmatic randomized control trial
}

Andrea K. Northwood ${ }^{1 *}$ D, Maria M. Vukovich', Alison Beckman ${ }^{1}$, Jeffrey P. Walter², Novia Josiah', Leora Hudak ${ }^{3}$, Kathleen O'Donnell Burrows ${ }^{1}$, James P. Letts ${ }^{4}$ and Christine C. Danner ${ }^{5}$

\begin{abstract}
Background: Despite an unparalleled global refugee crisis, there are almost no studies in primary care addressing real-world conditions and longer courses of treatment that are typical when resettled refugees present to their physician with critical psychosocial needs and complex symptoms. We studied the effects of a year of psychotherapy and case management in a primary care setting on common symptoms and functioning for Karen refugees (a newly arrived population in St Paul, Minnesota) with depression.
\end{abstract}

Methods: A pragmatic parallel-group randomized control trial was conducted at two primary care clinics with large resettled Karen refugee patient populations, with simple random allocation to 1 year of either: (1) intensive psychotherapy and case management (IPCM), or (2) care-as-usual (CAU). Eligibility criteria included Major Depression diagnosis determined by structured diagnostic clinical interview, Karen refugee, ages 18-65. IPCM $(n=112)$ received a year of psychotherapy and case management coordinated onsite between the case manager, psychotherapist, and primary care providers; CAU $(n=102)$ received care-as-usual from their primary care clinic, including behavioral health referrals and/or brief onsite interventions. Blinded assessors collected outcomes of mean changes in depression and anxiety symptoms (measured by Hopkins Symptom Checklist-25), PTSD symptoms (Posttraumatic Diagnostic Scale), pain (internally developed 5-item Pain Scale), and social functioning (internally developed 37-item instrument standardized on refugees) at baseline, 3, 6 and 12 months. After propensity score matching, data were analyzed with the intention-to-treat principle using repeated measures ANOVA with partial eta-squared estimates of effect size.

Results: Of 214 participants, 193 completed a baseline and follow up assessment (90.2\%). IPCM patients showed significant improvements in depression, PTSD, anxiety, and pain symptoms and in social functioning at all time points, with magnitude of improvement increasing over time. CAU patients did not show significant improvements. The largest mean differences observed between groups were in depression (difference, 5.5, 95\% Cl, 3.9 to $7.1, P<.001$ ) and basic needs/safety (difference, 5.4, 95\% Cl, 3.8 to $7.0, P<.001$ ).

Conclusions: Adult Karen refugees with depression benefited from intensive psychotherapy and case management coordinated and delivered under usual conditions in primary care. Intervention effects strengthened at each interval, suggesting robust recovery is possible.

Trial registration: clinicaltrials.gov Identifier: NCT03788408. Registered 20 Dec 2018. Retrospectively registered.

Keywords: Refugees, Depression, Primary care, PTSD, Basic needs, Case management

\footnotetext{
*Correspondence: anorthwood@cvt.org

${ }^{1}$ Center for Victims of Torture, 2356 University Ave W Ste 430, St Paul, MN

55114, USA

Full list of author information is available at the end of the article
}

(c) The Author(s). 2020 Open Access This article is distributed under the terms of the Creative Commons Attribution 4.0 International License (http://creativecommons.org/licenses/by/4.0/), which permits unrestricted use, distribution, and reproduction in any medium, provided you give appropriate credit to the original author(s) and the source, provide a link to the Creative Commons license, and indicate if changes were made. The Creative Commons Public Domain Dedication waiver (http://creativecommons.org/publicdomain/zero/1.0/) applies to the data made available in this article, unless otherwise stated. 


\section{Background}

The global refugee and migration crisis has reached unprecedented numbers, prompting the World Health Organization (WHO) to release a 2019-2023 Global Action Plan [1] that calls on health systems to adjust more rapidly to a new reality in which one out of seven persons worldwide is a migrant or refugee [2]. When refugees or migrants do receive health care, it is overwhelmingly in frontline settings such as humanitarian crisis arenas and primary care. Among other priorities, WHO's plan emphasizes the urgent need to mainstream refugee and migrant healthcare services, promote a range of short-term and long-term interventions, address the social determinants of health, and integrate mental health within refugee healthcare provision.

Refugee populations exposed to war and torture have elevated rates of depression, PTSD, anxiety, chronic pain, and acute medical conditions [3-7]. Karen refugees from Burma have high rates of torture and war trauma related to a 70year armed conflict with the government of Burma [8], and the health effects of these experiences have been compounded by lengthy stays in refugee camps on the ThaiBurma border without legal access to medical care, education, employment, or adequate food [9]. Newly resettled refugee populations present in primary care settings with a host of complex, interrelated biopsychosocial needs and profound access barriers involving culture, language, transportation, and health literacy [10-13]. These barriers, in concert with severe trauma and years of medical neglect preresettlement, create challenges for primary care clinics regarding elevated risk of severe disease, poor health outcomes, and high need for already limited clinic resources. Existing research [14-16] has described gaps in care for refugees navigating the U.S. medical system in particular.

While a strong body of evidence has supported the integration of behavioral health services into primary care to treat depression [17-21], anxiety [22, 23], and chronic health conditions $[24,25]$, no randomized control trials (RCTs) have investigated the efficacy of integrated services for refugee populations. Few trials exist in the field of refugee health due to the ethical and practical challenges of conducting experimental research with this population [26, 27]. Research has been constrained by small sample sizes, uneven comparison group sizes, singular outcomes, lack of control groups, lack of randomization, and non-blind assessment $[3,28]$.

RCTs are an essential component of establishing evidence of effectiveness. However, few RCTs resemble realworld clinical practice conditions or populations, which compromises both their applicability and credibility with practitioners [29-32]. For example, in a literature review of RCTs that examined representation of patients from "everyday clinical practice" in mental health, cardiology, and oncology, the authors conclude that a high proportion of the general disease population is excluded from trials, usually for reasons related to excluded patients' higher risk profile or co-morbidities [30]. Additionally, in RCTs of behavioral health interventions, length of treatment is often far shorter and breadth of outcomes assessed is narrower than it is in real-world psychotherapy practice [32]. There are no known RCTs of intensive patient-centered behavioral health treatments for refugees in primary care lasting longer than 6 months that address the broad array of presenting psychological and social problems that resettled refugees face. Patient-centered is defined here to mean the patient chooses goals to work on, preferred means of achieving goals (among options offered and guided by their psychotherapists and case managers), and the pace of self-directed change. Behavioral health services for refugees must be flexible and robust enough to address each individual's needs for psychosocial stabilization and each individual's trajectory in overcoming the debilitating psychological consequences that commonly follow catastrophic losses and traumatic events. Experienced refugee mental health providers are guided by a repository of general principles, knowledge, and skills in cross-cultural traumainformed practice [11, 26, 27, 33, 34] integrating various evidence-based components as opportunity and resources allow. There is dire need for pragmatic yet rigorous research on this type of real-world clinical model implemented in a primary care setting with refugee patients [35, 36].

We sought to examine the efficacy of behavioral health interventions located within the primary care setting that were congruent in length and flexibility to those provided in more specialist centres. We conducted a pragmatic RCT on a one-year behavioral health intervention consisting of psychotherapy and case management provided by refugee trauma specialists from the Center for Victims of Torture (CVT) within two urban primary care clinics serving Karen refugees from Burma, one of the largest refugee populations to arrive recently in our service area of Minnesota, USA [37].

The aim of the present study was to evaluate the benefits of intensive, coordinated psychotherapy and case management in primary care on common symptoms (depression, anxiety, PTSD, pain) and social functioning in refugees, relative to a comparison group who received care as usual from their primary care provider and usual referrals for mental health services.

\section{Methods}

\section{Study design and oversight}

A parallel-group randomized control trial was conducted with simple random allocation to either: 1) intensive psychotherapy and case management (IPCM) within the primary care clinic, or 2) care as usual (CAU), the clinic's usual process for managing mental health concerns 
including referring to community providers or providing brief, onsite behavioral health support.

Ethical trial conduct and safety were overseen and approved by the institutional review boards of the University of Minnesota, Healtheast, and the Minnesota Department of Human Services.

\section{Pragmatic design}

The growing call for more pragmatic studies [30] has led to increased analysis of their complexity. In any study, multiple components of design exist on continuums of highly pragmatic (i.e., Does the intervention work under usual conditions?) to highly explanatory (i.e., Does the intervention work under ideal, highly controlled conditions?) [38]. Accordingly, the key instrument framing conversations about pragmatic trials, the Pragmatic-Explanatory Continuum Indicator Summary (PRECIS-2) [39], uses a wheel format to capture a trial's scores on nine domains that affect external validity, on one end of the continuum, and isolation of precise causal mechanisms, on the other. These domains include how representative the study is of real-world conditions in participant eligibility criteria, recruitment path, care setting, organisation (expertise or resources required), flexibility in care delivery, flexibility in patient adherence, intensity of follow-up, relevance of primary outcomes to patients, and inclusiveness of the primary analyses [39]. Refugees with high trauma exposure, insecurity of basic needs and high mobility, complex physical and mental health presentations, and multiple healthcare access barriers make a compelling case for studies to be highly pragmatic in order to be applicable to primary care settings charged with their care.

The present study's design was pragmatic on seven of nine PRECIS-2 domains: (1) few eligibility criteria: adult Karen refugees from Burma, ages 18-65, with a diagnosis of Major Depressive Disorder (MDD); (2) recruitment occurred at the time of presentation and by primary care physician referral; (3) actual primary care setting; (4) providers had high flexibility in delivering the intervention of psychotherapy and case management according to patients' self-chosen treatment goals; (5) patients had complete flexibility in adherence to the intervention; (6) the primary outcomes were highly relevant to refugee patients, representing common presenting symptoms and areas of social functioning CVT clinicians routinely address; and (7) data analyses included all available data using intention to treat. The two domains yielding a less pragmatic score were organization (we used highly skilled refugee behavioral health providers) and follow-up (although patients in both groups were followed with the same intensity of measurement, IPCM patients received a greater number of visits than is typical of behavioral health services in a U.S. primary care setting). Overall the study was thus pragmatic except in two areas integral to our understanding of effective treatment for this vulnerable population.

\section{Patients, setting, and location}

Recruitment occurred on a rolling basis from May 2013 January 2017; data collection concluded in January 2018. Primary physicians at both clinics were aware of the parameters of the study as a randomized control trial for Karen refugee patients with depression and that eligibility (including current depression diagnosis) would be determined independently by a study clinician regardless of the patient's current or past diagnoses. Karen refugee patients were referred by their primary physician based on the presence of depression symptoms (including unremitting pain as a common manifestation of depression in refugees [3-7]) in two urban primary care clinics in St Paul, Minnesota, USA. Potential participants were invited to meet with a study clinician and a professional interpreter who explained the study and obtained informed consent. For those participants who requested time to consider whether they wanted to enroll, the study clinician made arrangements to follow up with them at a later date, usually a week later. A Consort flow chart [40] of patients through the study is presented in Fig. 1.

After obtaining informed consent, the clinician conducted an eligibility assessment comprising the major depressive episode section of the Structured Clinical Interview for DSM-IV (SCID) [41], psychosis screening questions, and the CAGE-AID [42] for substance use. Inclusion criteria were: Karen refugee, ages 18-65, meets criteria for MDD according to SCID interview (MDD criteria did not change in DSM-V). Exclusion criteria were: current enrollment in individual psychotherapy or mental health case management [43], active psychosis that study providers determined was not culturally derived or trauma-related (many patients had psychoticlike symptoms such as seeing shadows and ghosts that were normative cultural expressions of distress and these were not excluded), chemical dependency or reported problems with non-prescribed drugs or alcohol on the CAGE-AID, and acute need at the time of screening for a higher level of care than the study provided (e.g., inpatient treatment). Ineligible patients received alternative referrals, and the referring physician was informed by message in the electronic health record so that care as usual could proceed in a timely manner.

\section{Randomized allocation}

A coin toss by a research assistant otherwise uninvolved in the study was used to determine group allocation. Outcome assessors (research staff not involved in the intervention who administered the measures) were blind to group assignment.

\section{Intervention group}

IPCM patients received services from both a psychotherapist and a case manager for 1 year. Depending on patient availability, appointments were weekly or bi-weekly 


\section{Enrollment}

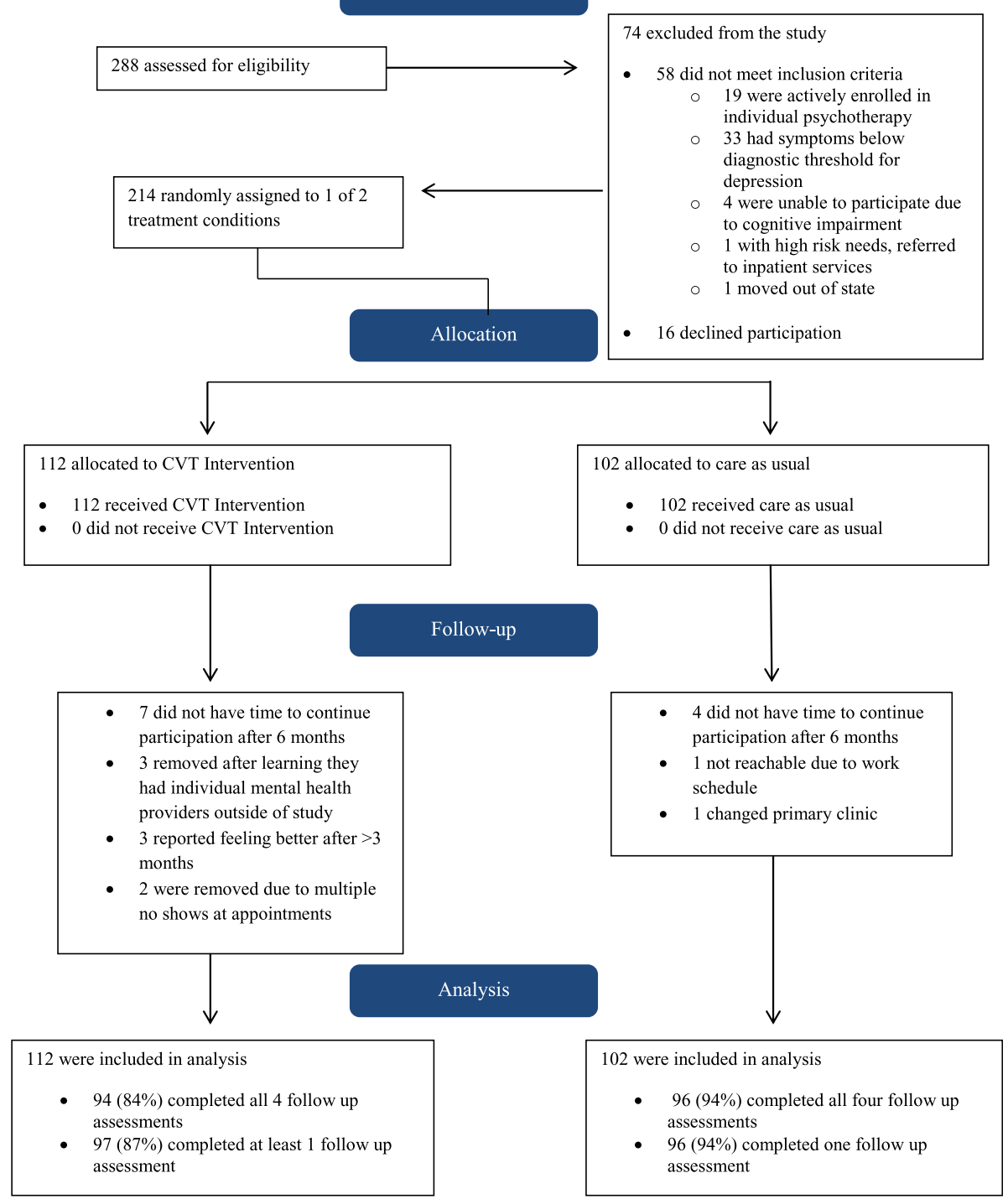

Fig. 1 Consort flow chart of Karen refugee patients through the RCT. Patients referred to the study and enrolled in the intervention or care-asusual groups from eligibility determination through baseline, 3, 6, and 12 months follow up

and lasted $45 \mathrm{~min}-1 \mathrm{~h}$. A professional interpreter was utilized unless the provider was a native Karen speaker.

CVT psychotherapists providing the intervention included 3 doctoral-level psychologists and 1 master-level clinical social worker. CVT case managers included 4 master-level social workers and 1 bachelor-level social worker. Additional training for clinical staff included: completion of a 10module blended learning course created by CVT called Fundamentals of Providing Services to Torture Survivors [44]; Adult Mental Health Targeted Case Management training provided by the state of Minnesota [45]; individual clinical supervision conducted by senior CVT psychotherapists; participation in monthly psychological consultation and clinical social work group supervision with other CVT providers, and up to $60 \mathrm{~h}$ of yearly continuing education training per individual provider.

Consistent with pragmatic randomized trial design to examine real-world practice with refugees [26, 29], providers delivering psychotherapy and case management tailored appropriate trauma and depression interventions to individual patients. Case management's function was to help patients gain access to medical, social, educational, vocational and other necessary services connected to their mental health needs [46]. Case management interventions focused on reestablishing safety and stabilization [33, 34, 47]; facilitating communication, problem-solving and understanding between patients and medical providers [48]; and increasing skill in navigating health and community systems in 
resettlement [48]. Each patient and his/her case manager developed and worked from an Individual and Community Support Plan (ICSP) [46] that prioritized 3-5 goals, stated in the patient's words (e.g., "I want to work to help my family with bills"; "I want to become U.S. citizen"). Core components of the case management are described in Table 1.

Psychotherapy functioned to increase patients' coping skills and understanding of their symptoms, as well as to alleviate these symptoms and their impact. Psychotherapists taught mind-body awareness and relaxation skills calibrated to survivors of severe trauma and catastrophic losses. They provided education about the connections between trauma/ stress and symptoms, use of medications and normative expectations for the doctor-patient relationship in Western culture (e.g., medications are not shared; dosage is not changed safely without consultation with one's doctor; patient is expected to raise concerns proactively rather than waiting to be asked, etc.), and compensatory strategies for patients with significant memory or concentration impairments to take

Table 1 Functions and Components of Psychotherapy and Case Management Intervention

\footnotetext{
Case Management

Function: assist patients to gain access to medical, social, educational, vocational and other necessary services connected to their mental health needs Components:

- Assessing patients' needs and goals and impact of mental illness, and incorporating patients' strengths and progress toward goals - Planning goals and goal-related steps, updating the individual and community support plan, finding new resources

- Referring and linking to resources, supports and services

- Coordinating with medical providers, community resources and natural supports identified by each patient as important to his or her recovery process

- Monitoring the effectiveness of the resources, supports and services being utilized, especially with respect to refugees navigating health and community systems in resettlement - Discussing the progress made toward goals

- Advocating as case managers on behalf of the patients' mental health needs with medical, legal and social systems

Psychotherapy

Function: increase patients' coping skills and understanding of their symptoms; alleviate symptoms and their impact Components:
- Facilitating mind-body awareness; teaching and practicing relax- ation skills
- Providing psychoeducation on the relationship between trauma/ stress and symptoms, treatment options for mental health symptoms, use of medications, and the doctor-patient relationship in Western medical culture
- Developing and teaching compensatory strategies for taking medications accurately and following health plan instructions that accommodate impairments in memory/concentration and other mental health symptoms
- Applying evidence-based trauma-focused treatments to reduce symptoms of depression, anxiety, and posttraumatic stress - Problem-solving with patients to decrease impact of symptoms and distress by changing coping behaviors and thought patterns - Advocating as psychotherapists on behalf of patients' mental health needs with medical, legal and social systems

their medications accurately and follow behavioral aspects of their health care plan. Psychotherapists applied evidencebased treatments for PTSD and depression tested on refugee populations, including Narrative Exposure Therapy and Cognitive Behavior Therapy $[3,28]$; they also utilised components of other psychoeducational approaches and traumafocused treatments, such as Sensorimotor Psychotherapy [49], and patient-centered methods such as Motivational Interviewing [50]. Where psychological assessment and diagnoses had implications for needed services or benefits, psychotherapists advocated within medical, legal, and social service systems on behalf of individual patient needs related to mental health symptoms (e.g., completing waiver forms for the U.S. civics exam and English language requirement for U.S. citizenship, etc.). Common components of the psychotherapy are summarized in Table 1.

At the team level, CVT's approach emphasized active interdisciplinary coordination and a relational focus anchored in cultural humility [51] to address survivors' priorities as the primary architects of their healing and work together to coconstruct meaning and behavioral change. CVT providers communicated frequently with one another and with patients' primary care providers to address overarching themes and challenges in a patient's care. CVT providers scanned their assessments and case notes into patients' Electronic Medical Records (EMRs). CVT clinicians also communicated with physicians via the EMR and reviewed their patients' EMRs with read-only access. When possible, clinicians communicated in-person with physicians spontaneously between patient sessions, in warm handoffs with patients, and in planned case consult meetings with physicians.

Trauma and loss were understood to have ongoing community-based sociopolitical and historical dimensions rather than being conceptualized as discrete past events that happened to individuals. Treatment was responsive to the instability and ongoing acculturation stressors in the lives of refugee patients requiring responses to multiple unplanned interruptions, including financial, housing, employment, family, and healthrelated crises. A Karen coordinator provided repeated, active follow-up to remind clients of appointments and overcome transportation and language barriers. Interventions addressed symptoms recognized by conventional biomedical culture and Karen idioms of distress identified by patients to their CVT providers. Common approaches used with the intervention group are described in a published toolkit for serving refugees in primary care settings [52].

\section{Control group}

Participants in the control group received care as usual, without CVT involvement beyond administration of outcome measures. Once randomized, CAU patients could be referred to a full range of behavioral health services 
by their primary care physician. Use of behavioral health services by patients in the CAU groups was monitored by primary care providers but not by the study.

\section{Data collection and measurement}

Demographic characteristics were collected prior to randomization. Pre-specified outcomes were mean change in depression, anxiety, PTSD, pain and social functioning scores over the year of enrollment. Outcomes were collected at baseline, 3, 6 and 12 months using instruments found to be reliable and valid with refugee populations. Presence and severity scores of symptoms associated with MDD and Generalized Anxiety were measured on a 4point Likert scale using the Hopkins Symptom Checklist25 (HSCL-25) [53]. Presence and severity of symptoms associated with PTSD were similarly measured using Part 3 (17 PTSD symptoms) of the Posttraumatic Diagnostic Scale (PDS) [54] adapted to assess DSM-V diagnostic criteria. Presence and severity of pain was measured using an internally developed 5-item Pain Scale with adequate internal consistency of $\alpha=.76$. Social functioning in meeting basic needs, stabilization, employment, social support, adjustment, and community engagement was measured with a 37-item standardized instrument on a 7-point Likert scale validated with refugees [55]. Instruments were selected based on extensive research indicating high prevalence of depression, anxiety, PTSD and pain in refugee populations $[3,56,57]$. Torture, war, and resettlement also impact social functioning, including basic needs, legal status, social support and involvement, employment and education, and engagement with one's geographic community. Measures were administered by a trained assessor, blinded to treatment condition, who followed scripted protocols and used a professional interpreter. Assessors had no contact with CVT providers to minimize breaches to blindness and bias. The only exception occurred when a participant expressed intent to harm self or others. In these instances, the protocol allowed for appropriate crisis response without breaching assessor blindness.

\section{Sample size}

Power analysis was originally conducted a priori using depression symptom scores as the outcome variable based on assumption of a $20 \%$ attrition rate [58]. Due to lower than expected attrition (10\%), we re-calculated a sample size of at least 95 in each treatment group (190 participants total) to detect statistical significance at the alpha $<.05$ level with power of $80 \%$ or greater.

\section{Analysis}

Mean (SD) baseline characteristics of participants randomized to the intervention or control groups were analyzed using t-tests for continuous and chi square tests for categorical data (see Table 2). Standardized t-scores were created for all outcomes using the normed population distribution collected at CVT [59]. All dependent variables met the statistical assumptions of normality, independence, homoscedasticity and sphericity prior to inferential analysis. Treatment effects were examined through repeated measures analysis of variance. Comparisons between groups were pre-specified and all tests were two-sided. Pairwise comparisons were performed post hoc with Sidak adjustment for comparison of mean scores at each time point between groups. All analyses were conducted according to intention-to-treat methods [60]. An alpha cutoff of $p \leq .05$ was used to assess statistical significance. Effect sizes were calculated using partial eta squared and interpreted as $0.010-.059=$ small, $0.060-.139=$ medium,$\geq 0.14=$ large [61]. Statistical analyses were conducted in Statistical Package for the Social Sciences (SPSS) 24 [62] and R version 3.4.4 [63].. As cases were allocated in a non-random way, weighing pragmatic concerns of case load, provider availability, and where possible gender matching, no analysis of provider-related differences was included in the analysis.

\section{Results}

Of the 288 patients screened for eligibility, 58 did not meet inclusion criteria and 16 eligible patients declined to participate. Of the 58 ineligible patients, 33 did not meet criteria for MDD; 19 were already receiving individual psychotherapy or case management; 4 were unable to participate in psychotherapy due to cognitive impairment; 1 required inpatient psychiatric care not available through the intervention; and 1 patient moved to another state.

Overall, 214 participants were enrolled in the study and completed a baseline assessment. Of these 187 (87.4\%) completed all four assessments; 193 (90.2\%) completed the baseline and at least one follow up assessment.

Participant characteristics at baseline by treatment group are provided in Table 2, including gender, age, employment status, completed years of education, reported experiences of torture and harm resulting from war trauma, household size and length of time spent resettled in the United States. Propensity score matching was performed using weighted regression to adjust for residual imbalances in length of resettlement between treatment groups [64]. No statistically significant differences were identified between groups in length of resettlement postmatching. All other demographic differences between treatment groups measured at baseline were nonsignificant. Therefore, no additional adjustments were made for potential confounding variables in the repeated measures analysis of variance.

On average, IPCM participants received $41.27 \pm 16.70$ psychotherapy sessions and $38.31 \pm 15.29$ case management sessions during their 1 year enrollment in the study. Average symptoms at baseline among all participants met clinical 
Table 2 Baseline Characteristics of Study Participants $(N=214)$

\begin{tabular}{|c|c|c|c|c|}
\hline & IPCM & $\mathrm{CAU}$ & Total Sample & $P$ \\
\hline Total- no. (\%) & $112(52.3)$ & $102(47.7)$ & $214(100)$ & \\
\hline Women- no. (\%) & $92(82.1)$ & $79(77.5)$ & $171(79.9)$ & \\
\hline $\mathrm{Age}^{\mathrm{a}}$ & $43.84 \pm 3.13$ & $41.77 \pm 3.91$ & $42.76 \pm 3.28$ & .247 \\
\hline Unemployed-no. (\%) & $94(83.9)$ & $88(86.3)$ & $182(85.0)$ & .607 \\
\hline Reported Torture-no. (\%) & $41(36.6)$ & $36(35.3)$ & $77(35.9)$ & .311 \\
\hline Reported Direct Harm-no. (\%) & 78 (69.6) & $66(64.7)$ & $144(67.3)$ & .194 \\
\hline Education Completed ${ }^{a}$ & $2.9 \pm 3.12$ & $2.6 \pm 3.67$ & $2.8 \pm 3.39$ & .519 \\
\hline Number in Household ${ }^{a}$ & $6.01 \pm 1.74$ & $6.24 \pm 2.02$ & $6.13 \pm 1.92$ & .372 \\
\hline Length of Resettlement ${ }^{a}$ & $4.11 \pm 2.5^{b}$ & $4.6 \pm 2.10^{b}$ & $4.29 \pm 2.34$ & .124 \\
\hline
\end{tabular}

aplus-minus values are means + standard deviations

${ }^{\mathrm{b}}$ Residual imbalances between groups in length of resettlement found in the raw data were adjusted for using propensity score matching with weighted regression

cutoffs (average raw item score $>1.75$ ) for depression and anxiety on the HSCL-25 [65] and were similarly elevated on the PDS. Outcomes in symptoms and social functioning over time are reported in Table 3.

\section{Intervention response}

Statistically significant changes in symptoms were found between groups, and the mean differences between groups were large (see Table 3). IPCM participants demonstrated statistically significant mean reductions in depression, anxiety, PTSD, and pain symptoms from baseline to 3 months. Positive treatment effects continued through 12 months in all symptom outcomes for the IPCM group. In contrast, CAU participants demonstrated non-significant reductions in symptom outcomes over time. Mean differences between groups were statistically significant in 3, 6 and 12 month outcomes of depression, anxiety and PTSD. Mean differences between groups for 3 and 6 month outcomes of pain were non-significant; however, statistically significant differences were observed between groups for 12 month outcomes of pain.

Statistically significant changes in social functioning outcomes were observed between groups, and the mean differences were large for basic needs/safety and cultural adjustment outcomes. Mean differences between groups for immigration stability, employment, social support and community engagement outcomes were moderate (see Table 3). Statistically significant mean differences between groups were observed from baseline to 12 months in basic needs/ safety, immigration stability, social support, cultural adjustment, and community engagement outcomes. Statistically significant mean improvements in basic needs/safety, social support, cultural adjustment and community engagement outcomes were observed for the IPCM group at each followup assessment. Non-significant mean differences were observed for immigration stability and employment outcomes between 6 to 12 months for the IPCM group. The CAU group demonstrated nonsignificant mean differences in all social functioning outcomes over time.

High rates of depression and PTSD are well documented among refugees. Incremental changes in the frequency of depression and PTSD symptoms over the length of the trial are depicted for each group in Fig. 2.

Safety/basic needs, employment and social support are critical aspects of resettlement. Incremental changes in the frequency of met social needs in safety, employment and social support over the length of the trial are illustrated in Fig. 3.

\section{Discussion}

In this pragmatic randomized control trial of 214 resettled adult Karen refugees with Major Depression receiving 1 year of psychotherapy and case management in a primary care clinic setting, patients demonstrated significant decreases in symptoms and significant increases in social functioning. These improvements were significant at 3 months, and additional positive treatment effects continued for the intervention group through the end of the intervention at 12 months, increasing in magnitude over time. Patients receiving care as usual, which potentially included behavioral health services provided onsite or in the community, did not significantly improve in reported symptoms or social functioning over 3,6 , and 12 months. The mean differences (effect sizes) observed between the intervention and care as usual groups were large for symptoms (depression, anxiety, PTSD, pain), meeting basic needs, and cultural adjustment; corresponding effect sizes were moderate for immigration stability, employment, social support, and community engagement.

To our knowledge, this study is the first of its kind in several respects. There have been no prior RCTs investigating the efficacy of behavioral healthcare integrated within primary care settings for refugees [35], much less RCTs that employ a pragmatic design to test an intervention that is congruent in length and flexibility with the care provided to refugees at specialist centres. These 
Table 3 Changes in symptoms and functioning over time between IPCM $(N=112)$ and CAU $(N=102)$

\begin{tabular}{|c|c|c|c|c|}
\hline & CAU t-score Means \pm SD & IPCM t-score Means \pm SD & Between-group Difference (95\% Cl) & Partial eta squared $^{a}$ \\
\hline \multicolumn{5}{|l|}{ Depression } \\
\hline Baseline & $52.78 \pm 5.25$ & $52.66 \pm 5.53$ & $.12[-1.34$ to 1.57$] \mathrm{NS}$ & \\
\hline 3 months & $52.03 \pm 5.79$ & $47.90 \pm 6.07^{*}$ & 4.13 [2.53 to 5.73$]^{* * *}$ & \\
\hline 6 months & $51.24 \pm 6.74$ & $46.87 \pm 6.24^{* * *}$ & $4.37[2.62 \text { to } 6.12]^{* * *}$ & \\
\hline 12 months & $51.42 \pm 5.51$ & $45.96 \pm 6.27^{* * *}$ & $5.47[3.87 \text { to } 7.06]^{* * *}$ & 0.214 \\
\hline \multicolumn{5}{|l|}{ Anxiety } \\
\hline Baseline & $52.29 \pm 3.65$ & $52.27 \pm 4.99$ & $.02[-1.21$ to 1.17$] \mathrm{NS}$ & \\
\hline 3 months & $52.12 \pm 6.49$ & $48.94 \pm 2.90^{* * *}$ & $3.18[1.85$ to 4.51$]]^{* * *}$ & \\
\hline 6 months & $51.14 \pm 3.78$ & $47.98 \pm 4.19^{* *}$ & $3.16[2.08$ to 4.24$]]^{* * *}$ & \\
\hline 12 months & $51.58 \pm 3.89$ & $46.91 \pm 2.85^{* * *}$ & $4.67[3.76$ to 5.58$] * * *$ & 0.193 \\
\hline \multicolumn{5}{|l|}{ PTSD } \\
\hline Baseline & $52.53 \pm 6.45$ & $52.90 \pm 6.13$ & $.37[-1.33$ to 2.07$] \mathrm{NS}$ & \\
\hline 3 months & $51.72 \pm 5.97$ & $48.80 \pm 6.02^{* * *}$ & $2.92[-1.30 \text { to } 4.54]^{* *}$ & \\
\hline 6 months & $50.75 \pm 5.46$ & $47.45 \pm 6.61^{* * *}$ & $3.30[1.66$ to 4.94$]]^{* *}$ & \\
\hline 12 months & $51.39 \pm 5.91$ & $46.76 \pm 6.52^{* * *}$ & $4.63[2.95$ to 6.31$]]^{* * *}$ & 0.224 \\
\hline \multicolumn{5}{|l|}{ Pain } \\
\hline Baseline & $52.05 \pm 9.67$ & $52.34 \pm 9.73$ & $.29[-2.33$ to 2.91$] \mathrm{NS}$ & \\
\hline 3 months & $51.85 \pm 9.32$ & $49.90 \pm 9.81^{*}$ & 1.9 [.63 to 4.53$] \mathrm{NS}$ & \\
\hline 6 months & $51.70 \pm 10.26$ & $49.68 \pm 9.45^{*}$ & 2.02 [.64 to 4.68$]$ NS & \\
\hline 12 months & $52.35 \pm 9.47$ & $48.56 \pm 10.51^{*}$ & $3.79[1.08 \text { to } 6.50]^{* *}$ & 0.172 \\
\hline \multicolumn{5}{|c|}{ Basic Needs/Safety } \\
\hline Baseline & $47.75 \pm 5.24$ & $48.06 \pm 5.52$ & $.31[-1.14$ to 1.76$] \mathrm{NS}$ & \\
\hline 3 months & $47.51 \pm 5.79$ & $51.54 \pm 6.06^{* *}$ & $4.03[2.43 \text { to } 5.63]^{* * *}$ & \\
\hline 6 months & $48.67 \pm 6.74$ & $51.72 \pm 6.25^{* * *}$ & $3.06[1.30$ to 4.82$] * * *$ & \\
\hline 12 months & $49.43 \pm 5.51$ & $52.63 \pm 6.28^{* * *}$ & $5.44[3.86 \text { to } 7.05]^{* * *}$ & 0.247 \\
\hline \multicolumn{5}{|c|}{ Immigration Stability } \\
\hline Baseline & $47.04 \pm 3.65$ & $47.42 \pm 4.57$ & $.04[-.73$ to 1.49$] \mathrm{NS}$ & \\
\hline 3 months & $46.78 \pm 6.49$ & $48.90 \pm 2.95^{*}$ & $2.21[.74$ to 3.50$] * * *$ & \\
\hline 6 months & $48.12 \pm 3.78$ & $50.36 \pm 4.21^{*}$ & 2.24 [1.16 to 3.32$] * * *$ & \\
\hline 12 months & $48.34 \pm 3.89$ & $50.67 \pm 2.91^{*}$ & $2.33[1.40 \text { to } 3.26]^{* * *}$ & 0.125 \\
\hline \multicolumn{5}{|l|}{ Employment } \\
\hline Baseline & $47.58 \pm 9.67$ & $48.12 \pm 9.74$ & $.54[-2.08$ to 3.16$] \mathrm{NS}$ & \\
\hline 3 months & $47.71 \pm 9.33$ & $50.69 \pm 9.81^{*}$ & $2.98[.40 \text { to } 5.56]^{* * *}$ & \\
\hline 6 months & $48.39 \pm 10.24$ & $51.83 \pm 9.49^{*}$ & $3.44[.77 \text { to } 6.11]^{* * *}$ & \\
\hline 12 months & $47.80 \pm 10.91$ & $52.36 \pm 10.45^{* *}$ & $4.56[1.68$ to 7.44$]]^{* *}$ & 0.131 \\
\hline \multicolumn{5}{|l|}{ Social Support } \\
\hline Baseline & $47.86 \pm 6.41$ & $47.05 \pm 6.17$ & $.81[-.88$ to 2.51$] \mathrm{NS}$ & \\
\hline 3 months & $49.51 \pm 5.97$ & $51.48 \pm 6.03^{*}$ & 2.37 [.75 to 3.99$]^{* *}$ & \\
\hline 6 months & $49.34 \pm 5.48$ & $51.39 \pm 6.61^{*}$ & 2.02 [.37 to 3.67$]^{*}$ & \\
\hline 12 months & $49.91 \pm 5.79$ & $51.84 \pm 6.53^{* * *}$ & $1.9[.26 \text { to } 3.60]^{*}$ & 0.133 \\
\hline \multicolumn{5}{|c|}{ Cultural Adjustment } \\
\hline Baseline & $48.70 \pm 5.09$ & $48.57 \pm 5.23$ & .13 [-1.26 to 1.52$] \mathrm{NS}$ & \\
\hline 3 months & $47.56 \pm 5.04$ & $51.29 \pm 5.31^{*}$ & $3.73[2.33 \text { to } 5.13]^{* * *}$ & \\
\hline 6 months & $49.47 \pm 5.52$ & $53.38 \pm 6.10^{* *}$ & $3.91[2.34 \text { to } 5.48]^{* * *}$ & \\
\hline
\end{tabular}


Table 3 Changes in symptoms and functioning over time between IPCM $(N=112)$ and CAU $(N=102)$ (Continued)

\begin{tabular}{|c|c|c|c|c|}
\hline & CAU t-score Means \pm SD & IPCM t-score Means \pm SD & Between-group Difference $(95 \% \mathrm{Cl})$ & Partial eta squared \\
\hline 12 months & $50.19 \pm 5.98$ & $54.27 \pm 6.02^{* * *}$ & $4.01[2.38 \text { to } 5.62]^{* * *}$ & 0.14 \\
\hline \multicolumn{5}{|c|}{ Community Engagement } \\
\hline Baseline & $48.52 \pm 8.27$ & $48.59 \pm 7.76$ & $.07[-2.23$ to 2.09$] \mathrm{NS}$ & \\
\hline 3 months & $48.94 \pm 8.02$ & $49.79 \pm 7.82^{*}$ & $.85[-1.29$ to 2.99$] \mathrm{NS}$ & \\
\hline 6 months & $49.91 \pm 7.89$ & $52.75 \pm 7.71^{* *}$ & $2.84[.73 \text { to } 4.94]^{* *}$ & \\
\hline 12 months & $50.13 \pm 8.26$ & $53.10+8.05^{* * *}$ & $2.97[.77 \text { to } 5.17]^{* *}$ & 0.138 \\
\hline
\end{tabular}

Abbreviation: NS Non significant

${ }^{*} P<.05$

*** $P<.01$

*** $P<.001$

${ }^{\mathrm{a}}$ Effect size, reported as partial eta squared values, indicates the size of the differences observed between groups. 0.01 or more are small effects, 0.06 or more are medium effects, and 0.14 or more are large effects

findings are important to the many specialized treatment centres operating in resettlement contexts including the United States, some of which offer services within hospital or primary care settings [66]. Prior research on behavioral health in refugees has focused on descriptive reports of symptomatology in response to pre- and postmigration stress and trauma [36]. Assessment of social functioning is rare, despite evidence that many protective factors for refugee health are social in nature [47, 67]. Clinical trials in refugee health are often limited by methodological weaknesses including small samples, nonrandom group assignment, non-blind assessment, and cross-sectional design; the most rigorous studies have evaluated a brief manualized treatment that would often be one component (e.g., cognitive processing therapy; narrative exposure therapy) of a more lengthy multidisciplinary treatment under real-world conditions (for recent examples, see [68-70]). This study has demonstrated that rigorous, pragmatic trials of behavioral health intervention can be effectively implemented in primary care to address the symptoms and functioning of refugee patients.
Primary care for refugees who have experienced trauma and catastrophic loss has presented unique challenges to physicians, particularly related to patient engagement, trust, and the treatment of chronic pain [11]. In this study, the delivery of intensive behavioral health services in the primary care clinic resulted in significant, sustained improvement across multiple areas of wellbeing. Our findings suggest that patients demonstrate greater improvements with more intensive psychotherapy and case management than is typically afforded in a primary care setting. Traditionally, integrated behavioral health services in primary care focus on brief assessment, brief treatment, and referral to other community based mental health services. This sample reflected common characteristics of refugee populations seeking medical care, as described in the literature: patients at intake reported high levels of torture and war trauma, posttrauma symptoms including pain, complex medical conditions, and unemployment. At baseline, patients reported substantial basic needs (food, shelter, housing) and low social support and cultural adjustment. And yet,

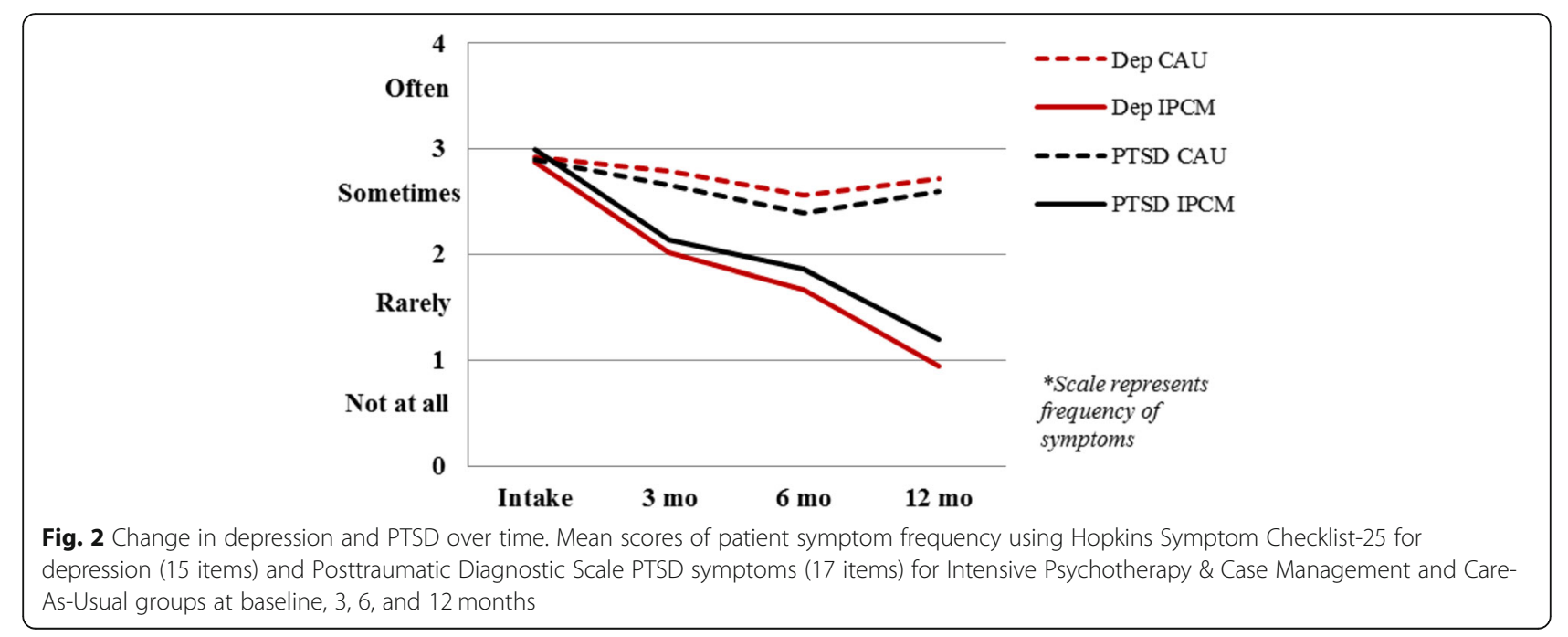




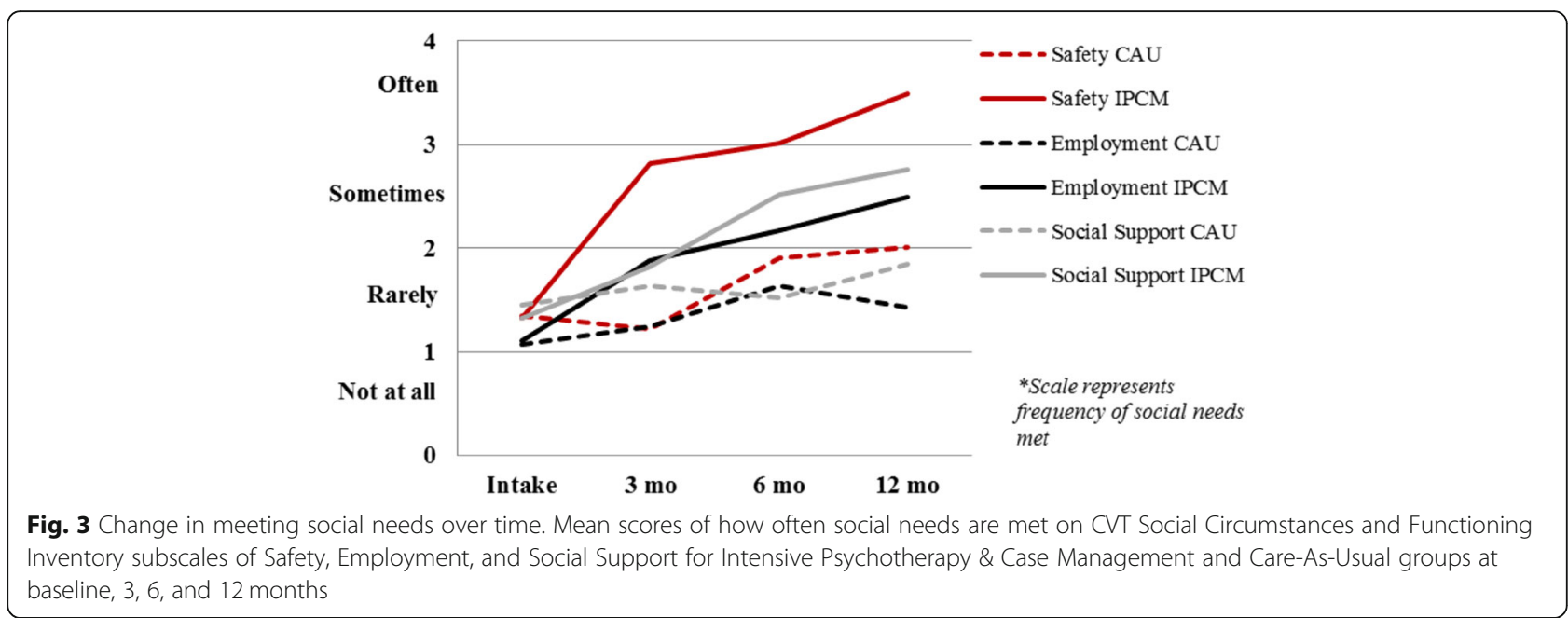

the steady and clinically meaningful improvement demonstrated by patients receiving the intensive intervention for 1 year showed that remarkable progress is possible with sufficient resources in place.

\section{Limitations}

Inherent in the design of a pragmatic RCT is the limitation with respect to isolating specific explanatory mechanisms [29]. This study does not examine which components of psychotherapy and case management were more strongly associated with improvements in symptoms and functioning. The amount, type and quality of nonCVT behavioral health interventions received by the care as usual group were not controlled for in the design.

While assessors were blind to study condition, primary care physicians and nurses could not be blinded as such, as coordination with a patient's primary care team was inherent to the intervention studied. It is possible that this knowledge of patients' conditions affected care in unknown ways, either biased for or against the intervention or care as usual.

Across conditions, most participants in this study were on multiple medications, including medications for depression, anxiety, sleep, and pain. This study was not resourced to measure prescription medications as taken, or not taken, or mis-taken, by refugees. Possible interactive effects of prescribed medications with this intervention, as well as traditional remedies used by refugee patients, will need to be explored in future research.

Given the paucity of controlled research with refugees, we chose in this study to focus on outcomes of symptom levels and adaptive functioning relevant to refugees that could be measured reliably by an assessor blinded to study condition. A limitation of the study is that we did not systematically measure other outcomes more tailored to the intervention group, such as types of goals developed under a patient-centered approach and the proportion that were met for the IPCM group. Results of a semi-structured interview that we administered to a subset of the IPCM group $(n=40)$ on active ingredients of the treatment from the patient's perspective are published elsewhere [48].

Regular comprehensive assessments by compassionate, skilled assessors may have influenced care and the study cannot explain why the care as usual group did not significantly improve in symptoms or functioning; it was not designed to systematically examine patterns of difference within the CAU group. In a busy real-world setting, some clients may have reported receiving mental health services when in fact they were merely completing regular measures. Physicians would have had to check the patient's electronic health record to verify this information and so may have been less likely to provide alternative referrals to a few CAU patients who wrongly reported receiving mental health services. Future studies parsing out what constitutes care as usual in refugee patients would do well to ensure via multiple channels that busy physicians are informed of study condition, tracking it, and not relying on patient report in a patient population with limited understanding of complex host-country services.

Other limitations of the study include gender imbalance and ethnic homogeneity. Eighty percent of participants were women. Gender differences and/or bias in reporting, assessing or treating mental health problems are discussed elsewhere [71] and may have been operative in this study. Although women and children are disproportionately represented in refugee populations [72], the findings may have less generalizability to men. For feasibility reasons, the study focused on one recently resettled refugee group: the Karen from Burma. Research with additional ethnic groups is needed to examine the efficacy of the intervention more broadly.

Finally, the benefits of not restricting treatment to a brief manualized protocol present corresponding challenges for replicability. We believe the value of examining principled, 
patient-centered services with delineatated functions and components by skilled refugee behavioral health providers exceeds the costs of not examining these non-manualized services and not making them more accessible to others through an evolving evidence base. Beyond the scope of this study, future research could examine a host of potential factors that might influence patient wellbeing or responsiveness to this type of intervention, including co-morbidities, prescribed medications, life events, other patient variables, and characteristics of the provider or intervention. Future pragmatic studies are needed to investigate the efficacy of this type of intensive intervention for a wider range of refugee patients in different care settings.

The implications for practice based on this study are that, despite the multidimensional complexities and challenges involved in their care, refugees can be well served by coordinated, intensive behavioral health interventions offered within the primary care setting. Primary care clinics serving large numbers of refugees can see better outcomes in these patients by offering intensive psychotherapy and case management services in the place of brief integrated behavioral health services or referral to community mental health.

\section{Conclusions}

The study conducted the first known randomized trial on the effectiveness of integrated behavioral health in primary care for refugees with Major Depression. Karen refugees receiving psychotherapy and case management over a 1 year period demonstrated mental health symptom reduction, pain reduction, and improvements in social functioning. Effects of the intervention were observed to strengthen at each measured interval, suggesting cumulative gains.

\section{Abbreviations \\ CAU: Care as usual; Cl: Confidence interval; CVT: Center for Victims of Torture; DSM: Diagnostic \& Statistical Manual of Mental Disorders; HSCL-25: Hopkins Symptom Checklist-25; ICSP: Individual and community support plan; IPCM: Intensive psychotherapy and case management; IRB: Institutional review board; MDD: Major Depressive Disorder; PDS: Posttraumatic Diagnostic Scale; PRECIS-2: Pragmatic-Explanatory Continuum Indicator Summary-2; PTSD: Posttraumatic Stress Disorder; RCT: Randomized control trial; SCID: Structured Clinical Interview for DSM-IV; SD: Standard deviation; SPSS: Statistical Package for the Social Sciences; WHO: World Health Organization}

\footnotetext{
Acknowledgments

Gregory Vinson, PhD (former CVT Research Associate) contributed to the concept and design of the study. Stanton Wood, MFA (CVT Strategic Initiatives Officer) helped secure funding. EhTa T. Zar, BA (CVT Client Services Coordinator) was essential in arranging transportation, interpretation, and scheduling for patients. Sonia Ausen-Anifani, MA and Robert Orazem, PhD (former CVT Research Assistants) served as blinded assessors; Alyce Eaton, BA (CVT Research Assistant) assisted with data entry and management. Craig Higson-Smith, MA (CVT Director of Research) provided invaluable assistance with the review of the manuscript. Many professional Karen-English interpreters were essential to communication with patients in all phases of the study. Finally, we thank the staff of the Roselawn and Bethesda Clinics and the 214 patients without whom this investigation would not be possible.
}

\section{Authors' contributions}

AKN and MMV had full access to all the data in the study and take responsibility for the integrity of the data and the accuracy of the data analysis. Concept and design: AKN; see also Acknowledgments. Clinicians administering the IPCM intervention: JPW, NJ, LH, KOB. Acquisition of data: AKN, MMV, AB, JPL, CCD. Analysis and interpretation of data: All authors. Clinicians administering the IPCM intervention did not have access to, or any knowledge of, the data until the study finished in January 2018. Statistical analysis: MMV. Drafting the manuscript: AKN, MMV. All authors have read and approved the final version of the paper.

\section{Funding}

The study was funded by the following: The Saint Paul Foundation, The F.R. Bigelow Foundation, Kresge Foundation, The Jacob and Valerie Langeloth Foundation, UCare Foundation, Medica Foundation, Greater Twin Cities United Way, Kinney Family Foundation, State of Minnesota Office of Justice Programs, The John and Ruth Huss Fund of the Saint Paul Foundation, Boston Scientific. The study was also conducted in partnership with HealthEast Care, University of Minnesota Physicians, and the Minnesota Department of Human Services. Study funders/supporters had no role in the design and conduct of the study; collection, management, analysis, and interpretation of the data; preparation, review, or approval of the manuscript; and decision to submit the manuscript for publication.

\section{Availability of data and materials}

The datasets collected and analyzed during the current study are not publicly available to maintain the privacy and confidentiality of participants enrolled in the trial and per health care system regulations of the trial sites. Data are available from the corresponding author on reasonable request and permission of the Center for Victims of Torture, University of Minnesota

Physicians Inc. and Healtheast Care systems.

Ethics approval and consent to participate

This study received ethics approval and oversight from the Healtheast Institutional Review Board (IRB) 1212002, University of Minnesota - Twin Cities IRB 1405 S50449 and the State of Minnesota Department of Human Services IRB 338. Written informed consent was obtained from all participants prior to enrollment in the trial.

\section{Consent for publication}

Not applicable.

\section{Competing interests}

The authors declare that they have no competing interests.

\section{Author details}

${ }^{1}$ Center for Victims of Torture, 2356 University Ave W Ste 430, St Paul, MN 55114 , USA. ${ }^{2}$ University of Minnesota, Boyton Health Mental Health Clinic, 410 Church St SE, Minneapolis, MN 55455, USA. ${ }^{3}$ Live-Oak, 1300 W Belmont Ave \#300, Chicago, IL 60657, USA. ${ }^{4}$ HealthEast Roselawn Clinic, 1983 Sloan PI \#1, St Paul, MN 55117, USA. ${ }^{5}$ St Joseph's Family Medicine Residency Program, University of Minnesota Physicians, Bethesda Family Medicine Clinic, 580 Rice St, St Paul, MN 55103, USA.

Received: 25 July 2019 Accepted: 20 January 2020

Published online: 28 January 2020

\section{References}

1. Director-General of World Health Organization. Promoting the health of refugees and migrants: draft global action plan 2019-2023. 2019. https:/apps.who.int/gb/ ebwha/pdf_files/WHA72/A72_25-en.pdf?ua=1. Accessed 21 May 2019.

2. World Health Organization: Refugee and migrant health. 2019. https://www. who.int/migrants/en. Accessed 21 May 2019.

3. Turrini G, Purgato M, Ballete F, Nosè M, Ostuzzi G, Barbui C. Common mental disorders in asylum seekers and refugees: umbrella review of prevalence and intervention studies. Int J Ment Heal Syst. 2017;11:51. https://doi.org/10.1186/s13033-017-0156-0.

4. Steel Z, Chey T, Silove D, Marnane C, Bryant RA, van Ommeren M. Association of torture and other potentially traumatic events with mental health outcomes among populations exposed to mass conflict and displacement: a systematic review and meta-analysis. JAMA. 2009;302(5):537-49. 
5. Dahl S, Dahl Cl, Sandvik L, Hauff E. Kronisk smerte hos traumatiserte flyktninger [chronic pain in traumatized refugees]. Tidsskr Nor Laegeforen. 2006;126:608-10.

6. Olsen DR, Montgomery E, Bøjholm S, Foldspang A. Prevalence of pain in the head, back and feet in refugees previously exposed to torture: a tenyear follow-up study. Disabil Rehabil. 2007;29:163-71.

7. Pfortmueller CA, Graf F, Tabbara M, Lindner G, Zimmermann H, Exadaktylos AK. Acute health problems in African refugees: ten years' experience in a Swiss emergency department. Wien Klin Wochenschr. 2012. https://doi.org/ 10.1007/s00508-012-0227-9.

8. Cook TL, Shannon PJ, Vinson GA, Letts JP, Dwee E. War trauma and torture experiences reported during public health screening of newly resettled Karen refugees: a qualitative study. BMC Int Health Hum Rights. 2015;15(1):8. https://doi.org/10.1186/s12914-015-0046-y.

9. Mitschke D, Mitschke AE, Slater H, Teboh C. Uncovering health and wellness needs of recently resettled Karen refugees from Burma. J Hum Behav Soc Environ. 2011;21(5):490-501.

10. Asgary R, Segar N. Barriers to health care access among refugee asylum seekers. J Health Care Poor Underserved. 2011;22(2):506. https://doi.org/10. 1353/hpu.2011.0047.

11. Crosby S. Primary care management of non-English-speaking refugees who have experienced trauma: a clinical review. JAMA. 2013;310(5):519-28.

12. Kreps GL, Sparks L. Meeting the health literacy needs of immigrant populations. Patient Educ Couns. 2008;71(3):328-32.

13. Karliner LS, Jacobs EA, Chen AH, Mutha S. Do professional interpreters improve clinical care for patients with limited English proficiency? A systematic review of the literature. Health Serv Res. 2007:42(2):727-54.

14. Pavlish CL, Noor S, Brandt J. Somali immigrant women and the American health care system: discordant beliefs, divergent expectations, and silent worries. Soc Sci Med. 2010;71(2):353-61.

15. Shannon $\mathrm{P}, \mathrm{O}^{\prime}$ Dougherty $M$, Mehta E. Refugees' perspectives on barriers to communication about trauma histories in primary care. Ment Health Fam Med. 2012;9(1):47-55.

16. Shannon PJ, Vinson GA, Cook TL, Lennon E. Characteristics of successful and unsuccessful mental health referrals of refugees. Admin Pol Ment Health. 2016;43(4):555-68

17. Björkelund C, Svenningsson I, Hange D, Udo C, Petersson E-L, Ariai N, et al Clinical effectiveness of care managers in collaborative care for patients with depression in Swedish primary health care: a pragmatic cluster randomized controlled trial. BMC Fam Pract. 2018;19(1):28. https://doi.org/ 10.1186/s12875-018-0711-z.

18. Butler M, Kane RL, McAlpine D, Kathol R, Fu SS, Hagedorn $H$, et al. Does integrated care improve treatment for depression? A systematic review. J Ambul Care Manage. 2011;34:113-25.

19. Kwan BM, Nease DE. The state of the evidence for integrated behavioral health in primary care. In: Talen MR, Burke Valeras A, editors. Integrated behavioral health in primary care: evaluating the evidence, identifying the essentials. New York: Springer; 2013. p. 65-98.

20. Ekers D, Murphy R, Archer J, Ebenezer C, Kemp D, Gilbody S. Nurse-delivered collaborative care for depression and long-term physical conditions: a systematic review and meta-analysis. J Affect Disord. 2013;149:14-22

21. Gilbody S, Bower P, Fletcher J, Richards D, Sutton AJ. Collaborative care for depression: a cummulative meta-analysis and review of longer-term outcomes. Arch Intern Med. 2006;166(21):2314-21.

22. Cape J, Whittington C, Buszewicz M, Wallace $P$, Underwood L. Brief psychological therapies for anxiety and depression in primary care: meta-analysis and metaregression. BMC Med. 2010;8(1):38. https:/doi.org/10.1186/1741-7015-8-38.

23. Muntingh $A D$, van der Feltz-Cornelis $C M$, van Marwijk HW, Spinhoven $P$, van Balkom AJ. Collaborative care for anxiety disorders in primary care: a systematic review and meta-analysis. BMC Fam Pract. 2016;17(1):62. https:// doi.org/10.1186/s12875-016-0466-3

24. Stewart JC, Perkins AJ, Callahan CM. Effect of collaborative care for depression on risk of cardiovascular events: data from the IMPACT randomized control trial. Psychosom Med. 2014;76:29-37.

25. Von Korff M, Katon WJ, Lin EH, Ciechanowski P, Peterson D, Ludman EJ, et al. Functional outcomes of multi-condition collaborative care and successful ageing: results of randomised trial. BMJ. 2011;343:d6612. https:// doi.org/10.1136/bmj.d6612.

26. Carlsson J, Sonne C, Silove D. From pioneers to scientists: challenges in establishing evidence-gathering models in torture and trauma mental health services for refugees. J Nerv Ment Dis. 2014;202(9):630-7.
27. Jaranson J, Quiroga J. Evaluating the services of torture rehabilitation programmes: history and recommendations. Torture. 2011;21:98-140.

28. Nosè M, Ballette F, Bighelli I, Turrini G, Purgato M, Tol W, et al. Psychosocial interventions for post-traumatic stress disorder in refugees and asylum seekers resettled in high-income countries: systematic review and metaanalysis. PLoS One. 2017;12:1. https://doi.org/10.1371/journal.pone.0171030.

29. Treweek S, Zwarenstein M. Making trials matter: pragmatic and explanatory trials and the problem of applicability. Trials. 2009;10(1):37. https://doi.org/ 10.1186/1745-6215-10-37.

30. Kennedy-Martin T, Curtis S, Faries D, Robinson S, Johnston J. A literature review on the representativeness of randomized control trial samples and implications for the external validity of trial results. Trials. 2015;16(1):495. https://doi.org/10.1186/s13063-015-1023-4.

31. Davis M, Balasubramanian BA, Waller E, Miller BF, Green LA, Cohen DJ. Integrating behavioral and physical health care in the real world: early lessons from advancing care together. J Am Board Fam Med. 2013;26(5):588-602.

32. Shean G. Limitations of randomized control designs in psychotherapy research. Adv Psychiatry. 2014. https://doi.org/10.1155/2014/561452 Accessed 15 Jul 2019.

33. Mollica RF. Healing invisible wounds: paths to hope and recovery in a violent world. Nashville: Vanderbilt University Press; 2006.

34. Center for Victims of Torture. Healing the hurt: a guide for developing services for torture survivors. 2005. https://www.healtorture.org/content/ healing-hurt. Accessed 22 Jul 2019.

35. Esala JJ, Vukovich MM, Hanbury A, Kashyap S, Joscelyne A. Collaborative care for refugees and torture survivors: key findings from the literature. Traumatology. 2018;24(3):168-85.

36. Pollard RQ, Betts WR, Carroll JK, Waxmonsky JA, Barnett S. deGruy FV III, et al. integrating primary care and behavioral health with four special populations: children with special needs, people with serious mental illness, refugees, and deaf people. Am Psychol. 2014;69(4):377-87.

37. Minnesota Department of Health. Primary refugee arrival data: Cummulative arrivals 1979-2017. 2018. https://www.health.state.mn.us/communities/rih/ stats/refcumm.pdf. Accessed 8 June 2019.

38. PRECIS-2 toolkit. https://www.precis-2.org/Help/Documentation/ ToolkitDownload. 2016. Accessed 21 May 2019.

39. Loudon K, Treweek S, Sullivan F, Donnan P, Thorpe KE, Zwarenstein M. The PRECIS-2 tool: designing trials that are fit for purpose. BMJ. 2015:350:h2147. https://doi.org/10.1136/bmj.h2147.

40. Schulz KF, Altman DG, Moher D. CONSORT 2010 statement: updated guidelines for reporting parallel group randomised trials. BMC Med. 2010; 8(1):18. https://doi.org/10.1186/1741-7015-8-18.

41. First MB, Spitzer RL, Gibbon M, Williams JB. Structured clinical interview for DSM-IV-TR Axis I disorders, research version, patient edition with psychotic screen (SCID-I/P W/ PSY SCREEN). New York: Biometrics Research, New York State Psychiatric Institute; 2002

42. Brown RL, Rounds LA. Conjoint screening questionnaires for alcohol and other drug abuse: criterion validity in a primary care practice. Wis Med J. 1995;94:135-40.

43. Minnesota statute 245.462 subdivision 3. 2018. https://www.revisor.mn.gov/ statutes/cite/245.462. Accessed 22 Jul 2019.

44. Fundamentals of providing services to torture survivors eLearning. https:// www.healtorture.org/content/fundamentals-providing-services-torturesurvivors-elearning. 2013. Accessed 6 Oct 2019.

45. Adult mental health-targeted case management. http://pathlore.dhs.mn. gov/courseware/AdultMentalHealth/TCM/Module_01/pages/0101.htm. 2019. Accessed 6 Oct 2019.

46. Minnesota Department of Human Services. Provider manual: adult mental health targeted case management and children's mental health targeted case management. 2018. https://www.dhs.state.mn.us/main/DHS16_167409. Accessed 5 June 2019.

47. Porter M, Haslam N. Predisplacement and postdisplacement factors associated with mental health of refugees and internally displaced persons: a meta-analysis. JAMA. 2005;294(5):602-12.

48. Esala J, Hudak L, Eaton A, Vukovich M. Integrated behavioral health care for Karen refugees: a qualitative exploration of active ingredients. Int J Migr Health Soc Care. 2018;14(2):133-45.

49. Ogden P, Fisher J. Sensorimotor psychotherapy: interventions for trauma and attachment (Norton series on interpersonal neurobiology). New York: WW Norton \& Co; 2015.

50. Miller WR, Rollnick S. Motivational interviewing: helping people change. 3rd ed. New York: Guilford Press; 2013. 
51. Foronda C, Baptiste DL, Reinholdt MM, Ousman K. Cultural humility: a concept analysis. J Transcult Nurs. 2016;27(3):210-7.

52. Beckman A, O'Donnell Burrows K, Walter J. Improving well-being for refugees in primary care: a toolkit for providers. St Paul, Minnesota: Center for Victims of Torture; 2019. https://healtorture.org/content/improving-wellbeing-refugees-primary-care-toolkit-providers. Accessed $22 \mathrm{Jul} 2019$

53. Derogatis LR, Lipmann RS, Rickels K. The Hopkins symptoms checklist (HSCL): a self-report inventory. Behav Sci. 1974;19:1-15.

54. Foa E, Cashman L, Jaycox L, Perry K. The validation of a self-report measure of PTSD: the posttraumatic diagnostic scale. Psychol Assess. 1997;9:445-51.

55. Vukovich M, Tracey H. The Social Circumstances and Social Functioning Inventory (SCFI-37): utility and validity in practice. Paper presented at 8th annual research symposium of National Consortium of Torture Treatment Programs. Washington D.C: National Consortium of Torture Treatment Programs; 2017

56. Williams AC, Peña CR, Rice AS. Persistent pain in survivors of torture: a cohort study. J Pain Symptom Manag. 2010;40(5):715-22.

57. Gureje O, Von Korff M, Simon GE, Gater R. Persistent pain and well-being: a World Health Organization study in primary care. JAMA. 1998;280(2):147-51.

58. Faul F, Erdfelder E, Lang A-G, Buchner A. G*power 3: a flexible statistical power analysis program for the social, behavioral, and biomedical sciences. Behav Res Methods. 2007:39:175-91.

59. Groth Marnet G, Wright AJ. Handbook of psychological assessment. 6th ed. New Jersey: John Wiley \& Sons; 2016

60. Heritier SR, Gebski VJ, Keech AC. Inclusion of patients in clinical trial analysis: the intention-to-treat principle. Med J Aust. 2003:179:438-40.

61. Cohen J. Statistical power analysis for the behavioral sciences. New York, NY: Routledge; 1988

62. Corportation IB. IBM SPSS Statistics (for Windows). Version 25.0. Amonk IBM Corp; 2017.

63. R Core Team. R: a language and environment for statistical computing. The $R$ project for statistical computing. http///www.R-project.org. Accessed 22 Jul 2019

64. Olmos A, Govindasamy P. A practical guide for using propensity score weighting in R. Practical Assessment, Research and Evaluation. 2015. http:// pareonline.net/getvn.asp?v=20\&n=13. Accessed 22 Jul 2019

65. Ventevogel P, Vries GJ, Scholte W, Shinwari NR, Faiz H, Nassery R, et al. Properties of the Hopkins symptom Checklist-25 (HSCL-25) and the selfreporting questionnaire (SRQ-20) as screening instruments used in primary care in Afghanistan. Soc Psychiatry Psychiatr Epidemiol. 2007:42:328-35.

66. Center for Victims of Torture. 2019. https://healtorture.org/content/ domestic-healing-centers. Accessed 22 Jul 2019.

67. Miller KE, Rasmussen A. The mental health of civilians displaced by armed conflict: an ecological model of refugee distress. Epidemiol Psychiatr Sci. 2017;26:129-38.

68. Ertl V, Pfeiffer A, Schauer E, Elbert T, Neuner F. Community-implemented trauma therapy for former child soldiers in northern Uganda: a randomized control trial. JAMA. 2011;306(5):503-12.

69. Bass JK, Annan J, Mclvor Murray S, Kaysen D, Griffiths S, Cetinoglu T, et al. Controlled trial of psychotherapy for Congolese survivors of sexual violence. N Engl J Med. 2013;368:2182-91.

70. Rahman A, Usman Hamdani S, Riaz Awan N, Bryant RA, Dawson KS, Firaz Khan $\mathrm{M}$, et al. Effect of a multicomponent behavioral intervention in adults impaired by psychological distress in a conflict-affected area of Pakistan: a randomized control trial. JAMA. 2016;316(24):2609-17.

71. World Health Organization Department of Mental Health and Substance Dependence. 2019. https//uww.who.int/mental health/media/en/242.pdf?ua=1. Accessed 6 Oct 2019

72. United Nations High Commissioner for Refugees. Global trends: forced displacement in 2017. 2018. https://www.unhcr.org/5b27be547.pdf. Accessed 22 July 2019

\section{Publisher's Note}

Springer Nature remains neutral with regard to jurisdictional claims in published maps and institutional affiliations.

Ready to submit your research? Choose BMC and benefit from:

- fast, convenient online submission

- thorough peer review by experienced researchers in your field

- rapid publication on acceptance

- support for research data, including large and complex data types

- gold Open Access which fosters wider collaboration and increased citations

- maximum visibility for your research: over $100 \mathrm{M}$ website views per year

At $\mathrm{BMC}$, research is always in progress.

Learn more biomedcentral.com/submissions 